\title{
Simulation and Optimization of a Sequenced Mixed-Model Line - Case Study of an Assembly of Gantry Car Washes
}

\author{
Lothar März ${ }^{1 *}$, Jörg Mielke \\ 1STREMLER AG, Lindau (Germany), *lothar.maerz@stremler.de \\ ${ }^{2}$ WashTec Cleaning Technology GmbH, Augsburg (Germany), jmielke@washtec.de
}

SNE 30(4), 2020, 159-164, DOI: 10.11128/sne.30.tn.10534 Received: July 12, 2020 (Selected ASIM SPL 2019 Postconf. Publ.); Revised: Oct. 8, 2020; Accepted: Oct. 20, 2020 SNE - Simulation Notes Europe, ARGESIM Publisher Vienna ISSN Print 2305-9974, Online 2306-0271, www.sne-journal.org

Abstract. In car wash machine manufacturing, the assembly line is characterized by product diversity and workforce flexibility. The main challenge of operational control is to schedule assembly tasks to workstations by taking workforce availability and qualification into consideration. In order to help planners to better analyse, plan, and evaluate the assembly line, in this paper, we develop a framework in which simulation and optimization are applied. Particularly, a simulation-based optimization approach is used to reduce excessive overload peaks for workstations. At last, an example of a solution for our industrial partner is given to show the feasibility and the applicability of our framework.

\section{Introduction}

Every company must face the challenge of offering top performance at globally competitive prices. In addition to technology and quality, customer service is playing an increasing role in all industries in order to differentiate their own range of services from those of their competitors. In order to successfully meet the requirements economically, it requires, on the one hand, a high level of innovation and strong technical competence. On the other hand, the long-term maintenance in global competition can only succeed, if the highly developed production skills are planned and controlled as flexible as possible, and, at the same time, at optimal cost.
Due to the labor-intensive production structure and the low level of automation, the employees in assembly lines of car wash manufacturers are still the main production resources. In order to keep the high German labor costs under control, a high degree of workforce flexibility is necessary.

\section{Workforce Planning in Sequenced Assembly Lines}

The aim of workforce planning is to manufacture the constantly changing production program with as few employees as possible [4]. Workforce flexibility is expressed, for example, by drifting across cycle times or switching workers from one station to another station within one cycle in order to avoid bottleneck situations (floaters). Drifting means that employees go to subsequent orders or follow the workpiece to the next station.

Floaters can be divided into internal and external floaters. External floaters are not planned in the line and support the employees of a station in the case of overloads in order to carry out the tasks involved within the specified cycle [1]. The employee balances the changing process time requirements over the cycle times. In the practice of deploying personnel in assembly, it also happens that employees perform activities at more than one station in one cycle. This always happens when there is enough free capacity at the main station to allow one of the assigned employees for working at another (floater) station. In this case, an internal floater balances the different process time requirements at the same cycle across two different stations. After performing the workload, the employee returns to his or her base station. 
Another factor influencing the balancing of the lines is the qualification of the employees. The planner is, therefore, faced with the daily task of carrying out the sequence of orders depending on the number of employees, the qualifications, and the process time requirements, to balance the process time requirements per cycle and station and to avoid both underutilization and overload cases.

In fact, due to the high process time spread of the products, it can be observed that there are often overload cases that could have been avoided by efficient sequence planning. The prerequisite for efficient planning is the exact predictability of the effects of employee behavior, taking into account the possibilities for work flexibility (drifting, jumping between stations, assigning employees to stations, etc.).

The simulation has established itself as state of the art to support the planning of assembly lines in recent years [5]. Simulation is used to make reliable predictions of the dynamic behavior of employees over the period of a delivery week. In order to predict the behavior of each employee, each individual process step is assigned to the employees and the process duration is calculated based on the qualification. The qualification considers the experience of the employee. This gives the planner a clear overview of the workflow depending on the order sequence.

However, the simulation only evaluates a planning scenario and does not determine an improvement in the planning solution. In this respect, the simulation is a very efficient tool for evaluating the effects of sequences on the workload of employees and the need for external floaters. The sequencing of a complex assembly line is a combinatorial optimization problem, which is unfortunately an NP-hard problem. To solve the real-world planning tasks under consideration of dynamic effects, classic solution algorithms or heuristics such as dispatching rules are insufficient. A promising approach to solve this task is simulation-based optimization [3].

The basic idea of simulation-based optimization is to simulate, evaluate, and compare different scenarios in order to create new scenarios using rule-based configuration. The basic idea of these iterations is the generation of feasible solutions. For example, the meta-heuristic method of genetic algorithms (GA) in combination with simulation or dispatch rules in combination with simulation have been successfully used several times in various industries to solve sequence problems $[2,6]$.
In addition to personnel planning and sequence optimization, the application presented in this article also serves to synchronize the line (Figure 1).

These three fields of application can be differentiated depending on planning horizon and objective: the workforce planning is based on a given sequence, and - in the case of a divergent personnel availability - it examines the effects and evaluates possible alternative personnel assignments. The sequence optimization runs in advance and determines minimum needs for employees and floaters. The balancing falls within the scope of workforce planning and distributes the processes to stations and employees in such a way that, despite the variety of variants, the workload of the employees is as uniform as possible.

This article focuses on sequence optimization. For this purpose, the scope of the illustration and the simulation of personnel deployment are first presented in Section 2. Section 3 explains the methodology for sequence optimization. Section 4 presents the results. The article closes with an outlook.

\begin{tabular}{|c|c|}
\hline $\begin{array}{l}\text { Workforce planning } \\
\text { - Impact of deviations of } \\
\text { personnel availability } \\
\text { - Realization of variations of } \\
\text { production programme } \\
\text { - Realization of variant peaks in } \\
\text { one shift to assign floaters }\end{array}$ & 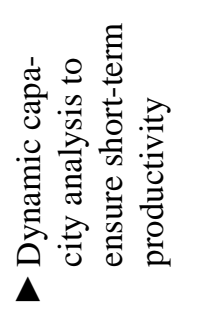 \\
\hline $\begin{array}{l}\text { Sequence optimization } \\
\text { - Minimizing overload cases per } \\
\text { cycle and station } \\
\text { - Adjustment of order sequence } \\
\text { on the basis of given criteria } \\
\text { - Determination of order volume }\end{array}$ & 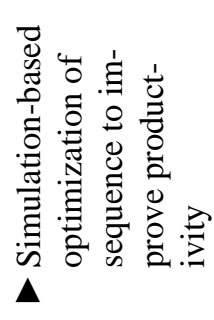 \\
\hline $\begin{array}{l}\text { Line Balancing } \\
\text { - Relocating of workload from } \\
\text { one station to another station } \\
\text { - Changing number of staff } \\
\text { - Adjustment of cycle time or } \\
\text { process times }\end{array}$ & 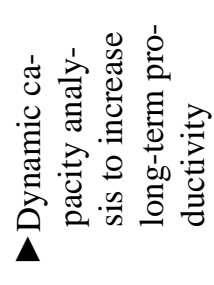 \\
\hline
\end{tabular}

Figure 1: Application areas of simulation-based line optimization. 


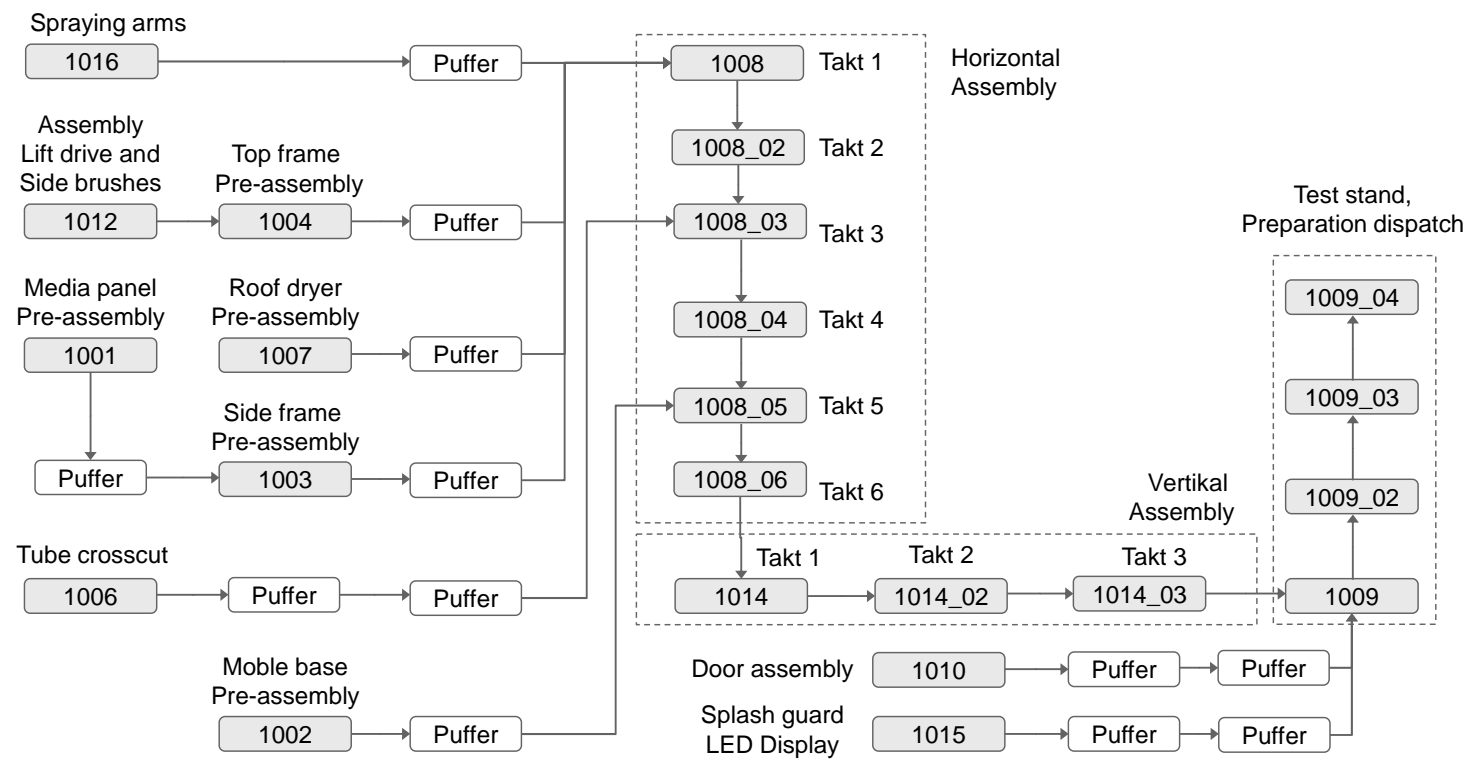

Figure 2: Scope of the sequenced assembly area.

\section{Assembly Structure and Personnel Deployment}

The sequenced production line at WashTec includes preassembly and final assembly stations. In total, the portal systems are assembled at a high rate of product diversity at 23 stations. The structure of the assembly is shown in Figure 2. The stations are run through in 17 clock cycles, which means that the pre-assembly of an order sometimes runs parallel to the main assembly work. The cycle time varies between 45 and 55 minutes depending on the order situation.

The employees are assigned to the stations and have station-dependent qualifications. A qualification of $100 \%$ means that the employee can fulfill the processes in the time specified in the work plan. If the employee has a qualification of $50 \%$, then he needs twice the time for the same job. The qualification levels of each employee for each station are stored in the employee qualification matrix.

The employees can drift. Drifting means that in the event of an overload of the subsequent order, the employees can go ahead (preparatory work) or rework in the next cycle. The drift capacity can be stored in the simulation model depending on the cycle time per station. For example, a drift capacity of +0.5 cycle times means that the employees assigned to this station can rework for a total of 25 minutes at a given cycle time of 50 minutes, for example.
Employees can also act as floaters. In the practice of deploying personnel in assembly, it happens that employees perform activities at more than one station in one cycle. This happens whenever there is enough free capacity at the home station to allow one of the assigned employees for working at another (floater) station. The potential floater employee must be identified as such in the personnel deployment table in advance of planning.

There are also floaters who are not assigned to the stations and who step in whenever the core workforce can no longer meet the process time requirements within their time frame.

Figure 3 shows an example of the process time requirements for each cycle as the left bar per group. To its right, the bars represent the workload of the employees per cycle, with the number of bars showing the number of employees. The $100 \%$-line marks the capacity of the employees considering their qualifications.
Workload and utilization of staff per cycle Cycle $1-7$ in $\%$ of capacity

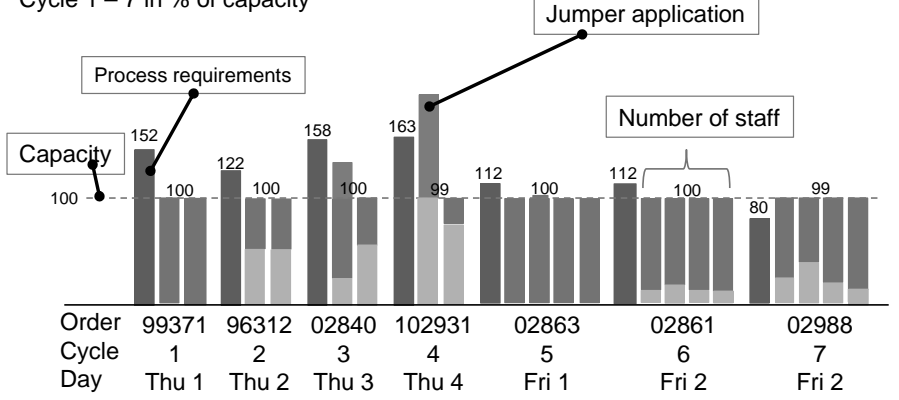

Figure 3: Process time requirements and personnel deployment. 
This shows to what extent the pending orders exceed the capacity of the assigned employees per cycle. The light sections of the bars show which process time portions of the previous cycle had to be processed in the subsequent cycle (rework). The bars above the capacity line of $100 \%$ show that an (external) floater was necessary in this cycle. It is important to avoid these cases. By continuous improvements of the data quality and of line balancing, the number of external floaters could be minimized to a few events per shift.

\section{Architecture and Methodology of Sequence Optimization}

The architecture of the application is structured based on the functions that are specified by the planning process. After reading the order and process time data from SAP, the initial sequence is first simulated. The assignment of work content to the stations is based on the work plan data from SAP, in which the individual process times are assigned to the stations. This first sequence is based on an upstream, rough division of orders into shifts under consideration of main features. This prevents predictable overload cases from occurring too often in one shift due to optional equipment options. This first sequence proposal serves as a reference for assessing the subsequent sequence optimizations. The sequence optimization considers a production period of two weeks, which means a total number of about 100 products. Each product is an individual configuration based on a product platform (lotsize 1).

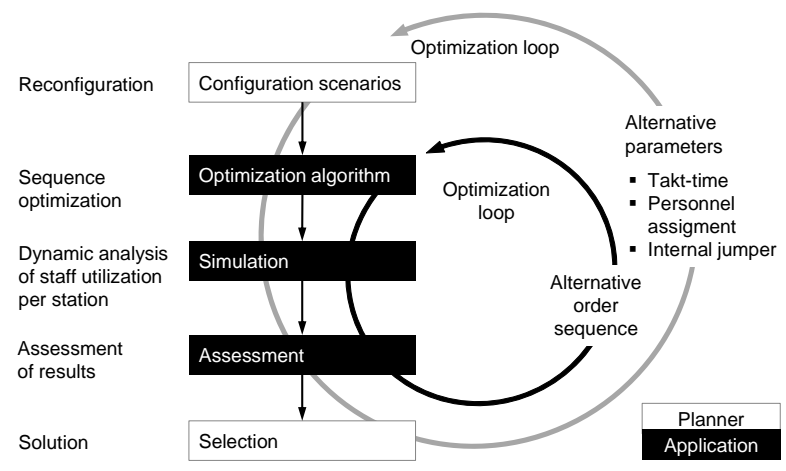

Figure 4: Optimization process.

The evaluation takes place based on the floater operating times. For this purpose, all floater operating times are summed up across all stations and all cycles.
Figure 4 shows the iterative run of the sequence optimization integrated in the higher-level process for optimal configuration.

The operation is as follows: After simulation and evaluation of the initial sequence, alternative sequences are determined and simulated again. The selected optimization algorithm determines the selection of new order sequences. The results of the modified orders are evaluated and compared. After a given number of iterations, which depends on an abort criterion, it is clear which floater operations are necessary. If the result is not satisfactory, the dispatcher can make alternative configurations. A few parameters are available to him, for example:

- Increase or decrease the cycle time

- Changed personnel assignment to stations,

e.g., by assigning less qualified employees to

stations with underload

- Classification of employees as internal floaters

Afterwards, the system-supported sequence optimization can run again. In the case of changes on very short term, which, e.g., may occur before start of work if the planned personnel availability is not given, the dispatcher can solve critical bottleneck situations by changing personnel assignments. Prior to a shift, sequence optimization can help to find an optimal sequence in combination with the other configuration options.

The objective function of the optimization tries to minimize the usage times of floaters. After each simulation run, the number of employments and the total floater operating times per station are determined. The shorter the employment time, the better the result. Table 1 shows the results of the simulation with the floater operations per station.

The optimization was initially tackled by using rulebased algorithms. After selecting a time period of, e.g., a calendar week, the algorithm goes through the following steps:

1. Sort the stations in descending order according to their floater operating times

2. Take the station with the highest floater deployment time

3. Sort the orders pending at this station in the period under review by process time totals

4. Sort the orders alternately according to the highest and lowest value of the total accumulated process times, i.e., first take the order with the highest process time requirements, then the order with the lowest process time requirements, then the order with the second-highest ones, etc. 
5. Continue in this scheme until all orders have been placed in the sequence

6. Simulate the sequence and save the result

7. Take the next station from the sorted list from Step 1

8. Continue until all stations with a floater assignment greater than zero have been rated

9. Compare the results and choose the scenario with the lowest floater operating times

\begin{tabular}{|c|c|c|c|c|c|}
\hline Station & $\begin{array}{l}\text { Floater } \\
\text { Rate } \\
\text { [\%] }\end{array}$ & $\begin{array}{l}\text { Floater } \\
\text { Rate } \\
\text { [Minutes] }\end{array}$ & $\begin{array}{l}\text { Quan- } \\
\text { tity }\end{array}$ & Factor & $\begin{array}{l}\text { Weighted } \\
\text { Quantity }\end{array}$ \\
\hline 1001 & 22 & 875.5 & 16 & 1 & 16 \\
\hline 1012 & 22 & 311.6 & 13 & 1 & 13 \\
\hline 1007 & 12 & 350 & 9 & 1 & 9 \\
\hline 1016 & 6 & 101 & 2 & 1 & 2 \\
\hline 1003 & 5 & 243 & 7 & 1 & 7 \\
\hline 1004 & 0 & 0 & 0 & 1 & 0 \\
\hline 1006 & 20 & 261.2 & 10 & 1 & 10 \\
\hline 1008 & 0 & 0 & 0 & 1 & 0 \\
\hline 1008_02 & 0 & 0 & 0 & 1 & 0 \\
\hline 1002 & 1 & 41 & 1 & 1 & 1 \\
\hline 1008_03 & 16 & 232 & 9 & 1 & 9 \\
\hline 1008_04 & 0 & 0 & 0 & 2 & 0 \\
\hline 1008_05 & 0 & 0 & 0 & 2 & 0 \\
\hline 1008_06 & 0 & 0 & 0 & 2 & 0 \\
\hline 1010 & 24 & $1,022.5$ & 11 & 2 & 22 \\
\hline
\end{tabular}

Table 1: Evaluation of the floater deployments per simulation run.

The number of floater employments can also be used as an evaluation instead of the floaters' duty times. A weighting factor (Figure 5) that takes this into account has been introduced, because the floater operations are more difficult in the final assembly stations.

\section{Sequence Optimization Results}

Overall, with the rule-based sequence optimization improvements in the use of floaters, based on the total number of employees in the line, between $3 \%$ and $5 \%$ could be achieved.

Table 2 shows the results of a total of six randomly selected weeks, which were sorted in ascending order depending on the employment times of the floater.
The floater operating times of the initial sequence are compared with the results of the rule-based optimization.

\begin{tabular}{lllllll}
\hline $\begin{array}{l}\text { Floater } \\
\text { time [min] }\end{array}$ & $\mathbf{1}$ & $\mathbf{2}$ & $\mathbf{3}$ & $\mathbf{4}$ & $\mathbf{5}$ & $\mathbf{6}$ \\
\hline Initial seq. & 3,102 & 6,272 & 6,891 & 9,660 & 9,781 & 12,996 \\
\hline $\begin{array}{l}\text { Optimiza- } \\
\text { tion }\end{array}$ & 2,804 & 3,845 & 4,336 & 5,853 & 6,333 & 10,076 \\
\hline $\begin{array}{l}\text { Improve- } \\
\text { ment [\%] }\end{array}$ & 9.6 & 38.7 & 37.1 & 39.4 & 35.3 & 22.5 \\
\hline
\end{tabular}

Table 2: Sequence optimization results.

The results show that the maximum improvement can be achieved if the sum of the floater operating times is in a certain range.

The employment of floaters is the result of the product mix. In the ideal range, improvements of up to $40 \%$ less deployment times of floaters could be shown! However, due to smaller sequence periods (e.g., 2-3 days or shifts) and improved line balancing, smaller improvement potentials can also be expected.

The simulation was carried out in the self-developed, high-performance simulation and optimization platform STREMLER REALTIME TECHNOLOGIES. The simulation takes less than a second to simulate the assembly of a shift, making the results of an optimization run available within 70 seconds - including data import and export - via an interface to the database.

In a further step, heuristic methods are now to be used in parallel in order to determine further optimization solutions depending on the available planning time.

\section{Outlook}

With the introduction of the application for simulationbased personnel planning and sequence optimization in the assembly area, WashTec Cleaning Technology $\mathrm{GmbH}$ is treading the path towards a supply chain in real time.

The application for simulation-based optimization of the sequenced assembly line, which has been running for over a year, is based on a modular planning architecture that enables the successive introduction of functionalities to plan and optimize the value chain.

The full benefit of this modular principle can be generated with the implementation of a consistent mapping of the value chain in the planning modules and a customer-specific defined supply chain platform. 
These are mainly based on the following relationships:

- Continuous adjustment of production to the market development by pull from shipping date and a prompt reaction of the value chain (production close to the market)

- Higher planning security and harmony in production through simulation-based dynamic capacity analyzes and optimization, considering all relevant influencing factors in production

- Takt-based synchronisation of planning and control of the value chain steps

- Continuous tracking of the weighted targets of productivity, service, and profitability

- Higher productivity through optimal sequencing and better lot sizes in all stages

Optimized planning and scheduling of assembly represents a complex planning case at WashTec. This new step enables significant improvements in productivity and service as well as drastic savings in planning effort.

\section{References}

[1] Becker C, Scholl A. A Survey on problem and methods in generalized assembly line balancing. European Journal of Operational Research. 2006; 168: 694-715.

[2] Jósvai J. Optimierungsmethoden der Reihenfolgeplanung mit Hilfe von Simulation. In: Dangelmaier W, Laroque C, Klaas A, editors. Simulation in Produktion und Logistik. Paderborn: HNI-Verlagsschriftenreihe; 2013; pp. 71-76.

[3] Law A, McComas M. Simulation-based optimization. In: Yücesan E, Chen CH, Snowdon JL, Charnes JM, editors. Proceedings of the 2002 Winter Simulation Conference. Piscataway, New Jersey: IEEE; 2002. pp. 41-44.

[4] März L, Tutsch H, Auer S, Sihn W. Integrated production program and human resource allocation planning of sequenced production lines with simulated assessment. In: Dangelmaier W, Blecken A, Delius R, Klöpfer SW, editors. Advanced Manufacturing and Sustainable Logistics. Berlin, Heidelberg: Springer; 2010. pp.408-418.

[5] Pinedo M. Planning and scheduling in manufacturing and service. $3^{\text {rd }}$ ed. New York: NY: Springer; 2007.

[6] Werner F. Genetic algorithm for shop scheduling problems: A survey. Preprint Series. 2011; 11: 1-66. 